\title{
Reply-Letter to the Editor: What to Do, and What Not to Do, When Diagnosing and Treating Breakthrough Cancer Pain (BTcP): Expert Opinion
}

\author{
Working Group Nientemale DEI $\cdot$ R. Vellucci ${ }^{1}$ (D) G. Fanelli $^{2} \cdot$ P. A. Cortesi ${ }^{3}$ • \\ R. Pannuti ${ }^{4}$ C. Peruselli ${ }^{5}$ P. Romualdi ${ }^{6}$
}

Published online: 20 June 2016

(c) The Author(s) 2016. This article is published with open access at Springerlink.com

Dear Editor,

We would like to thank Raffaele Giusti and colleagues [1] for their comments on our paper [2].

Consistent with the motto "Actions speak louder than words", we have gathered a group of opinion leaders in palliative care and pain treatment who, for several years, have been committed to treat breakthrough cancer pain (BTcP). Our experience, gained by close contact with cancer pain sufferers, has allowed us to explore the literature on $\mathrm{BTcP}$ and to transfer it into clinical practice. We are convinced that the situation in Italy [3, 4], in Europe [5] and in other countries [6], is far from encouraging.

In Italy, the phenomenon of under-treatment of cancer patients [7] and $\mathrm{BTcP}[3,4,8]$ is still an important problem.

It must be made clear that rapid-onset opioids (ROOs) do not seem to be used very often in clinical practice [5, 8], as can be indirectly deduced from the Italian sales data.

R. Vellucci

renato.vellucci@gmail.com

1 SOD Cure Palliative e Terapia del Dolore, Ospedale Universitario Careggi, Florence, Italy

2 SC Anestesia, Rianimazione e Terapia Antalgica, Azienda Ospedaliero-Universitaria di Parma, Parma, Italy

3 Research Centre on Public Health (CESP), University of Milan-Bicocca, Monza, Italy

4 Fondazione ANT Italia Onlus, Andria, Italy

5 SC Cure Palliative, Ospedale di Biella, Ponderano, BI, Italy

6 Dipartimento di Farmacia e Biotecnologie, Alma mater studiorum, Università di Bologna, Bologna, Italy
Specifically, about one-third of patients with BTcP did not receive any kind of rescue therapy, even in cases of patients with at least three attacks per day [8]. In terms of the drugs prescribed, one-third received a World Health Organization level I drug, while among the opioids, immediate-release (IR) morphine is significantly more frequently administered (71.2\%) than oral transmucosal fentanyl citrate (18.3\%) [8]. Even if IR morphine and fentanyl both act through the same mechanism of action, the route of administration influences the bioavailability of the drug, and the lipophilic future of fentanyl allows it to reach the brain very quickly.

At first sight, IR morphine might appear to be less expensive, but it has a pharmacokinetic profile, which is inappropriate for most episodes of $\mathrm{BTcP}$ and becomes more efficacious than placebo after only $45 \mathrm{~min}$, postbaseline [9].

That is not to say that morphine does not have a role in the treatment of BTcP, but rather that it may be useful in the management of breakthrough pain episodes lasting for more than $60 \mathrm{~min}$, and may be considered in the preemptive management of volitional incident pain or procedural pain [10].

One fact seems unequivocal: missing or inappropriate treatment of $\mathrm{BTcP}$ results in an increase of $\mathrm{BTcP}-$ related office visits, emergency department visits, and hospitalizations [11-14], often with long hospital stays, and oncologic restaging, all of which have a significant impact on costs, much more relevant than the costs related to using ROOs in place of IR morphine. On the contrary, the correct utilisation of ROOs in clinical practice can reduce the burden of BTcP. Better analgesic efficacy can be translated into savings in health-care resources as well as an improved quality of life for patients [11]. Nevertheless, 
further studies are needed to better quantify the economic impact of BTcP and the benefits of using ROOs instead of IR morphine.

We think that the long-term viability of our health-care system is closely linked to our clinical choices.

We are fully aware that BTcP exacts a significant toll on patients, their families, caregivers, and social networks, the health-care system, and society at large. To lessen these effects, we have prepared a decalogue of "Five Things to Do and Five Things Not to Do" in the diagnosis and treatment of BTcP; but add that this is what we do every day with patients in clinical practice. We are convinced that this approach represents a solid basis for making the necessary progress for clinical management of $\mathrm{BTcP}$, making our job easier.

Furthermore, considering the variable approach on cancer pain management in the different Italian regions, depending on the environment and local resource availability, we considered it essential and more useful to share and discuss opinions between a consistent number of palliative care experts from all over the country and define some shared methods, rather than working on standard recommendations. In addition, we also paid close attention to communication with patients and their caregivers, an important issue often underestimated.

An interesting issue needing further reflection is that, in our opinion, there is not a direct correlation between the number of papers written about BTcP management and awareness of the medical profession, and we believe in the importance of continuing to draw attention to the diagnosis and treatment of BTcP.

Moreover, the presence of several papers clearly suggests that the debate is open.

Nevertheless, there is, of course, nothing that cannot be improved further, that is why a new publication, a fortiori, if esteemed valid as you consider ours, should be seen as an opportunity. However, we recognised that continuous publications of new consensus, expert opinions, systematic reviews and guidelines, without strong critical appraisal, could not help policy makers, clinicians, and most of all cancer patients. For this reason, in the future, fewer papers but created with the involvement of a larger group of experts who are representative of all experts involved in the management of BTcP and with different approaches and experiences, could be more useful to guide the decision on the treatment and management of BTcP.

Compliance with Ethical Standards Renato Vellucci declares no financial, personal and commercial disclosures. He had academic relationships with pharmaceutical industries (Grunenthal, Molteni, Norgine and italfarmaco) for intellectual scientific activities, participation to scientific boards, invitations to Meetings as speaker. Paolo Cortesi has received speaking honoraria from Angelini.
Funding All authors declare no funding was received.

Conflict of interest Guido Fanelli, Carlo Leruselli, Raffaela Pannuti and Patrizia Romualdi declare no conflict of interest.

Open Access This article is distributed under the terms of the Creative Commons Attribution-NonCommercial 4.0 International License (http://creativecommons.org/licenses/by-nc/4.0/), which permits any noncommercial use, distribution, and reproduction in any medium, provided you give appropriate credit to the original author(s) and the source, provide a link to the Creative Commons license, and indicate if changes were made.

\section{References}

1. Giusti R, Verna L, Iacono D et al. Comment on "What to Do, and What Not to Do, When Diagnosing and Treating Breakthrough Cancer Pain (BTcP): Expert Opinion”. Drugs. 2016;76. doi: $10.1007 / \mathrm{s} 40265-016-0593-0$

2. Working Group Nientemale DEI, Vellucci R, Fanelli $G$ et al. What to Do, and What Not to Do, When Diagnosing and Treating Breakthrough Cancer Pain (BTcP): Expert Opinion. Drugs. 2016;76(3):315-330

3. Apolone G, Corli O, Caraceni A, Negri E, Deandrea S, Montanari M. Greco MT; Cancer Pain Outcome Research Study Group (CPOR SG) Investigators. Pattern and quality of care of cancer pain management. Results from the Cancer Pain Outcome Research Study Group. Br J Cancer. 2009;100(10):1566-74.

4. Mercadante S, Costanzo BV, Fusco F, Buttà V, Vitrano V, Casuccio A. Breakthrough pain in advanced cancer patients followed at home: a longitudinal study. J Pain Symptom Manage. 2009:38(4):554-60.

5. Davies A, Buchanan A, Zeppetella G, Porta-Sales J, Likar R, Weismayr W, SlamaO, Korhonen T, Filbet M, Poulain P, Mystakidou K, Ardavanis A, O'Brien T,Wilkinson P, Caraceni A, Zucco F, Zuurmond W, Andersen S, Damkier A, Vejlgaard T, Nauck F, Radbruch L, Sjolund KF, Stenberg M. Breakthrough cancer pain: an observational study of 1000 European oncology patients. J Pain Symptom Manag.2013;46(5):619-28.

6. Bedard G, Davies A, McDonald R, Hawley P, Buchanan A, Popovic M, Wong E, Chow E. Breakthrough cancer pain: a comparison of surveys with European and Canadian patients. Support Care Cancer. 2015;23(3):791-6.

7. Deandrea S, Montanari M, Moja L, Apolone G. Prevalence of under-treatment in cancer pain. A review of published literature. Ann Oncol. 2008;19(12):1985-91.

8. Greco MT, Corli O, Montanari M, Deandrea S, Zagonel V. Apolone G; Writing Protocol Committee; Cancer Pain Outcome Research Study Group (CPOR SG) Investigators. Epidemiology and pattern of care of breakthrough cancer pain in a longitudinal sample of cancer patients: results from the Cancer Pain Outcome Research Study Group. Clin J Pain. 2011;27(1):9-18.

9. Zeppetella G, Davies A, Eijgelshoven I, Jansen JP. A network meta-analysis of the efficacy of opioid analgesics for the management of breakthrough cancer pain episodes. J Pain Symptom Manag. 2014;47(4):772-785.e5.

10. Davies AN, Dickman A, Reid C, Stevens AM, Zeppetella G; Science Committee of the Association for Palliative Medicine of Great Britain and Ireland. The management of cancer-related breakthrough pain: recommendations of a task group of the Science Committee of the Association for Palliative Medicine of Great Britain and Ireland. Eur J Pain. 2009;13(4):331-8. 
11. Paolo A. Cortesi, Flavia Kheiraoui, Giuseppe Casale, Carlo Favaretti, Giovanni Guarrera, Pierangelo Lora Aprile, Patrizio Piacentini, Lorenzo G Mantovani. Budget impact analysis del fentanyl citrato sublinguale nella gestione del breakthrough cancer pain. 2015;4(3). http://www.ijph.it/pdf/99/014.pdf. Accessed 30 Apr 2016.

12. Abernethy AP, Wheeler JL, Fortner BV. A health economic model of breakthrough pain. Am J Manag Care. 2008;14(5 Suppl 1):S129-40.
13. Kuo KL, Saokaew S, Stenehjem DD. The pharmacoeconomics of breakthrough cancer pain. J Pain Palliat Care Pharmacother. 2013;27(2):167-75.

14. Vellucci R, Mediati RD, Gasperoni S, Piergallini LM, Valentino MC, Bruno GM, Di Matteo S, Colombo GL. Pharmacoeconomic considerations about breakthrough cancer pain. Value Health. 2015;18(7):A665-6. 